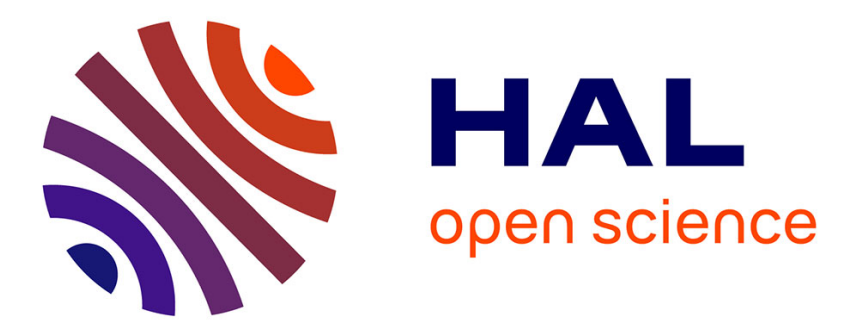

\title{
Dynamic control of DHM for ergonomic assessments
} Giovanni de Magistris, Alain Micaelli, Paul Evrard, Claude Andriot, Jonathan Savin, Clarisse Gaudez, Jacques Marsot

\section{To cite this version:}

Giovanni de Magistris, Alain Micaelli, Paul Evrard, Claude Andriot, Jonathan Savin, et al.. Dynamic control of DHM for ergonomic assessments. International Journal of Industrial Ergonomics, 2013, 43, pp.170-180. 10.1016/j.ergon.2013.01.003 . hal-00972324

\section{HAL Id: hal-00972324 https://hal.science/hal-00972324}

Submitted on 3 Apr 2014

HAL is a multi-disciplinary open access archive for the deposit and dissemination of scientific research documents, whether they are published or not. The documents may come from teaching and research institutions in France or abroad, or from public or private research centers.
L'archive ouverte pluridisciplinaire HAL, est destinée au dépôt et à la diffusion de documents scientifiques de niveau recherche, publiés ou non, émanant des établissements d'enseignement et de recherche français ou étrangers, des laboratoires publics ou privés. 


\title{
Dynamic control of DHM for ergonomic assessments
} Giovanni De Magistris
a,*
, Alain Micaelli ${ }^{\mathrm{a}}$, Paul Evrard $^{\mathrm{a}}$, Claude Andriot $^{\mathrm{a}}$, Jonathan Savin $^{\mathrm{b}}$, Clarisse $_{\text {Marsot }^{\mathrm{b}}}$

${ }^{a}$ CEA, LIST, Interactive Simulation Laboratory, 18 route du panorama, BP6, Fontenay aux Roses, F- 92265 France

${ }^{b}$ Institut national de recherche et de sécurité (INRS), rue du Morvan, CS 60027, Vandouvre-lès-Nancy, F- 54519 France

\begin{abstract}
Physical risk factors assessment is usually conducted by analysing postures and forces implemented by the operator during a work-task performance. A basic analysis can rely on questionnaires and video analysis, but more accurate comprehensive analysis generally requires complex expensive instrumentation, which may hamper movement task performance.

In recent years, it has become possible to study the ergonomic aspects of a workstation from the initial design process, by using digital human model (DHM) software packages such as Pro/ENGINEER Manikin, JACK, RAMSIS or CATIA-DELMIA Human. However, a number of limitations concerning the use of DHM have been identified, for example biomechanical approximations, static calculation, description of the probable future situation or statistical data on human performance characteristics. Furthermore, the most common DHM used in the design process are controlled through inverse kinematic techniques, which may not be suitable for all situations to be simulated.

A dynamic DHM automatically controlled in force and acceleration would therefore be an important contribution to analysing ergonomic aspects, especially when it comes to movement, applied forces and joint torques evaluation. Such a DHM would fill the gap between measurements made on the operator performing the task and simulations made using a static DHM.

In this paper, we introduce the principles of a new autonomous dynamic DHM, then describe an application and validation case based on an industrial assembly task adapted and implemented in the laboratory. An ergonomic assessment of both the real task and the simulation was conducted based on analysing the operator/manikin's joint angles and applied force in accordance with machinery safety standards (Standard NF EN ISO 1005-1 to 5 and OCcupational Repetitive Actions (OCRA) index). Given minimum description parameters of the task and subject, our DHM provides a simulation whose ergonomic assessment agrees with experimental evaluation.
\end{abstract}

Keywords: Digital Human Model, Dynamic control, Ergonomic analysis, Virtual reality

\section{Introduction}

Work-related musculoskeletal disorders (WRMSD) represent a major proportion of registered and/or compensatable work-related diseases in many countries, (Sjogaard et al., 1995)(Bernard, 1997), particularly those involving the lower back, neck, shoulder, forearm and wrist.

Several studies have identified a relationship between "work-related" diseases and physical risk factors at work (Bernard, 1997)(Kao, 2003). Job physical characteristics frequently quoted as WRMSD risk factors, based on experimental science and epidemiologic investigations, include rapid and repetitive motion patterns, heavy lifting, forceful manual exertions or prolonged static postures. As a result, safety standards have gradually detailed those issues and physical risk factor assessment at the earliest design stages has become a concern for industrial companies.

*Corresponding author. Tel: +3301 46547450 Email address: giovanni.de-magistris@cea.fr (G. De Magistris)
A basic analysis of a task can rely on questionnaires, interviews and video analysis. Besides, numerous indicators can be used for physical risk assessment of job activities, usually specific to a body part or a type of activity: commonly encountered assessment methodologies include RULA (MacAtamney and Cortlett, 1993) for the upper limbs, REBA (Hignett and MacAtamney, 2000) for the whole body, NIOSH equations, standards such as NF EN 1005 - Part2 (AFNOR, 2003) for load handling or NF EN 1005 - Part 5 (AFNOR, 2007) for repetitive tasks (this standard is based on the OCcupational Repetitive Action (OCRA) method (Occhipinti, 1998)). A more accurate and comprehensive analysis requires collection of exertion (force sensor and/or electromyography) and posture data (e.g. motion capture technique) from operators performing similar tasks. This analysis entails complex expensive instrumentation that may hamper task performance.

When designing a new task, an alternative is to use digital human models (DHM) for ergonomic analysis. For example, Sub-section 4.2 .5 of EN 1005-4 states that 
DHM can be used for anthropometry and postures assessment (AFNOR, 2008). Using DHM, it is currently possible to study, analyze and visualize complex movements or postures in a user-friendly three-dimensional graphical interface. Hence, an initial level of future workstation ergonomic assessment is possible even at the early stages of the design process without the need for direct measurements on human subjects (Chaffin, 2005)(Gomes et al., 1999)(Dukic et al., 2007). Examples of DHM software packages used for ergonomic assessment are Pro/ENGINEER Manikin Analysis, SAMMIE (Porter et al., 2004), JACK (Badler, 1997), CATIA-HUMAN Design and Analysis tools, RAMSIS and SANTOS.

However the simulations computed with these software packages usually rely on kinematics animation frameworks. Such frameworks use either pre-recorded motions, obtained through tracking system and motion capture, or interactive manual positioning of the DHM body through mouse, menus and keyboard. In the first case, simulations are realistic but they require extensive instrumentation of a full scale mock-up of the future workstation or a similar existing one. They are extremely time consuming because of motion capture data processing (Bradwell and Li, 2008). Furthermore, their ability to predict complex human postures and movements for various sizes and dimensions in a timely and realistic manner is strictly dependent on the accuracy of the motion database. In the second case, simulations are fairly subjective (the designer, possibly with no specific ergonomic skill, chooses arbitrarily a posture or trajectory). Again, they are time consuming (built up like a cartoon) or usually appear unnatural (Chaffin, 2007), even though they possess semi-automatic controls provided by a set of behaviours such as gazing, reaching, walking and grasping. These issues do not encourage designers to consider alternative scenarios, which would be beneficial for a comprehensive assessment of the future work situation. Moreover, such software packages are subject to numerous limitations: restriction to static models and calculation, neglect of balance or posture maintaining exertion. Neither do they consider contact forces between the DHM and objects (at best the designer has to arbitrarily set the contact force magnitude and direction manually). For these reasons, assessment of biomechanical risk factors, based on simulations of industrial or experimental situations, may lead to real stress underestimation up to 40-50\% (Lamkull et al., 2009) (Savin, 2011).

A challenging aim therefore consists in developing a virtual human model capable of computing automatic, dynamic, realistic movements and internal characteristics (position, velocities, accelerations and torques) in quasireal time, based on a simple description of the future work task, to achieve realistic ergonomics assessments of various work task scenarii at an early stage of the design process.

This type of dynamic DHM controlled in acceleration and force using simulated physics, forms the crux of our research. In our simulation framework, the entire motion of the human model in the virtual environment is dictated by real-world Newtonian physical and mechanical simulation, along with automatic control of applied forces and torques. We focus on repetitive tasks of the upper limb in this paper, which introduces our virtual human model and demonstrates its possibilities for physical risk evaluation at the design stage. The first and the second parts of the paper describe the application case used as an experimental and validation frame, then outline the principles of our dynamic DHM controls. The third part describes its outcomes, especially comparison of assessments based on real and simulated data. Finally, in the fourth part, we discuss the issues raised by the approach and its prospects.

\section{Human Subject Experiment}

\subsection{Experimental task description}

The research frame focuses on repetitive activities that present a significant risk of upper limb WRMSD. Our case study deals with the task of insert fitting. This activity comes from the automotive industry but can also be found in electric household appliance or other industries, in which the constant product evolution results in frequent, quick changes to production lines, requiring multiple manual operations. Insert fitting here consists in clipping small metal parts to the plastic instrument panel of a vehicle prior to screwing components to the panel.

\subsection{Subjects}

Our application case was first studied during an ergonomic assistance assignment conducted by France's Institut national de recherche et de sécurité (INRS), Laboratory for Biomechanics and Ergonomics (Gaudez, 2008). The same team subsequently adapted it to laboratory experimentation in accordance with biomedical research requirements. Eleven healthy right-handed subjects (nine males and two females) took part in the study [age $=29.4$ \pm 9.2 yrs (mean \pm standard deviation), height $=177.7 \pm$ $10.3 \mathrm{~cm}$, body mass $=75.9 \pm 9.3 \mathrm{~kg}]$. The subjects gave their informed consent to the experiments, which were approved by the institutional ethics committee, and completed a health questionnaire. The subject's anthropometry was measured to build human subject models based on the Hanavan model (Hanavan, 1964) (size, weight and 41 body segment measurements).

\subsection{Apparatus}

The workstation comprised a force platform assembled on a lift table fitted with a row of ten insert supports arranged at $45^{\circ}$ from back to front in the sagittal plane.

A ten camera Motion Analysis System was implemented to record the whole body positions and postures. A single top view camera, synchronized with the motion capture system, was used to record the subject's activity: these recordings could subsequently be used to view the real movements and correct for marker losses or hidden movements. 

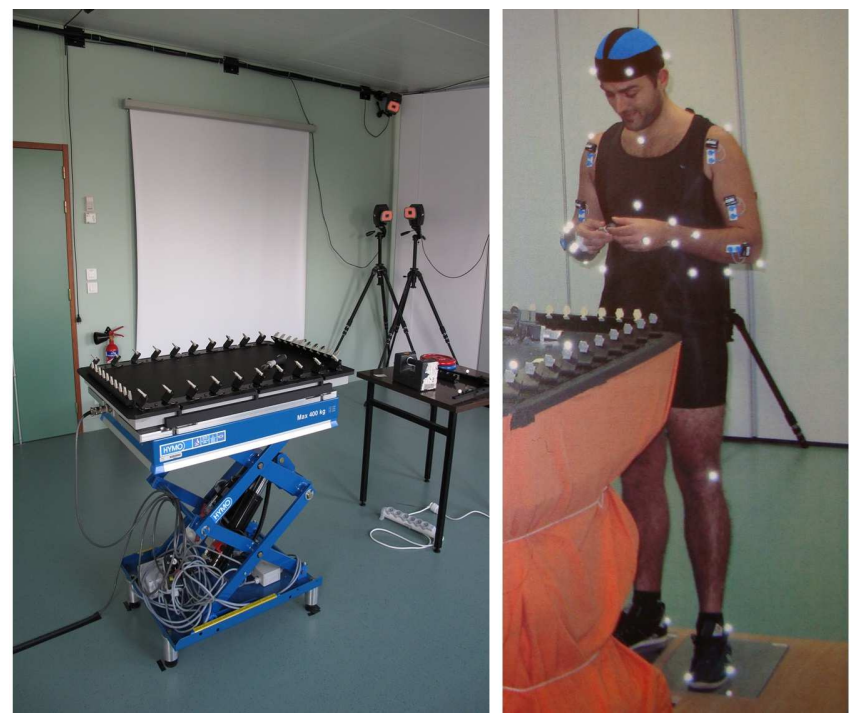

Figure 1: Actual work task at the left and human subject experiment at the right

The external force exerted during the activity was measured by the force platform attached to the table (AMTI, model BP600900-1000). This recorded both forces and moments in three spatial planes.

Data were recorded from the Motion Analysis System and the force platform simultaneously at a $100 \mathrm{~Hz}$ sampling rate.

\subsection{Experimental procedure}

Subjects were asked to perform the experimental task according to the two methods used in the workshop: either using only fingers or using a hand-held tool meeting specific ergonomic criteria (NST-n168, 1998). Each time, they fitted ten inserts into the ten parallel supports at constant pace (one insert every 4 seconds). The two methods were performed in random order.

The height of the table was adapted to the subject's anthropometry. It was set to $90 \%$ of elbow-ground height, in accordance with European standards for a standing work activity requiring normal vision and precision (CEN, 2008).

\subsection{Data analysis}

The fitting of the first and last two inserts of the row were recorded but not processed: only insertion of the six central clips was retained for characterizing an average task, forming a basis for simulation and ergonomic assessment (postures, applied forces and cycle time).

Motion capture data were post-processed to retrieve the subject anatomical joint angles (shoulder flexion/extension, abduction/adduction and internal/external rotation, elbow pronation/supination, etc.) and to make ergonomic assessment of the experimental task: motion capture markers positions were first filtered $(25 \mathrm{~Hz} 4 \mathrm{th}$ order Butterworth low pass), then processed to calculate joint angles using the Calcium solver module (MarquardtLevenberg optimization algorithm) of the Cortex suite (3.0.0 software version).

\subsection{Experimental observations}

All subjects implemented the same strategy when using the hand-held tool: they all picked up ten inserts with the left hand and placed them with this hand on the top of the tool, which was held in the right hand. They then pushed them onto the support using only the right hand. Yet, subjects implemented various strategies when using only their fingers:

1. Six subjects picked up ten inserts one at a time and clipped them onto the support using only the right hand

2. Four subjects picked up the inserts from the table with the left hand, then transferred them to the right hand, which was then used to clip them onto the support

3. One subject picked up the inserts from the table with the right hand, transferred them to the left to position them properly, then transferred them back to the right, which was then used to clip them onto the support. We excluded this last strategy from our study

Using the hand-held tool, the average applied force during insertion was $F x=-15 N, F y=-50 N, F z=40 N$. For the two fingered insertion variants, this force was $F x=-25 N, F y=-60 N, F z=55 N$. These values have been used as parameters of our simulations.

\subsection{Ergonomic assessment}

Our research, focusing on highly repetitive assembly activities with a high risk of upper limb MSD, prompted us to apply the OCRA index (Occhipinti, 1998). This index is currently recommended by European Standard EN 1005-5, supporting European Directive 2006/42/EC, the so-called Machinery Directive (EU, 2006). The latter directive requires designers to perform a priori risk assessment of their product, machine or workstation at the initial stage to ensure the lowest possible level of risk in relation to the state of the art.

The OCRA index provides an assessment of workrelated musculoskeletal disorder risk factors associated with repetitive movements of the upper limbs. This concise exposure index is the ratio between the number of actions actually performed by the upper limbs (ATA) during repetitive tasks and the corresponding number of recommended actions (RTA) for each upper limb. The OCRA index is given by the formula:

$$
\text { OCRA index }=\frac{\text { ATA }}{\text { RTA }}
$$

ATA is equal to:

$$
\mathrm{ATA}=F \cdot D=(\mathrm{NTC} \cdot 60) / C T \cdot D
$$


$F$ is the frequency per minute, NTC is the number of technical actions in a cycle, $C T$ is the cycle time in seconds, and $D$ is the evaluated net duration of the repetitive task during the work shift in minutes.

RTA is equal to:

$$
\begin{aligned}
& \mathrm{RTA}=\mathrm{RPA} \cdot\left(R c_{M} \cdot D u_{M}\right) \\
& =C F \cdot P o_{M} \cdot R e_{M} \cdot A d_{M} \cdot F o_{M} \cdot D \cdot\left(R c_{M} \cdot D u_{M}\right)
\end{aligned}
$$

RPA is the partial reference number of technical action for task; $C F$ is the frequency constant of technical actions per minute, used as a reference (30 actions per minute); the other multiplying factors, with scores ranging between 0 and 1 , depend on posture $\left(P o_{M}\right)$, repetitiveness $\left(R e_{M}\right)$, additional $\left(A d_{M}\right)$ risk factors (vibrating tools, exposure to cold or refrigeration, etc.), force $\left(F o_{M}\right)$, recovery $\left(R c_{M}\right)$, selected based on lack of recovery behavior and duration $\left(D u_{M}\right)$.

Table 1 is used to assess the risk. Level 1 (no risk) indicates that the condition examined is fully acceptable, the level 2 (low risk) indicates that the exposure may be significant and careful monitoring for induced health effects should be introduced (health surveillance). Level 3 (risk) indicates that the exposure is definitely significant and the higher the value, the higher the risk. In this case, actions should be undertaken to improve working conditions, along with close monitoring for induced effects.

\begin{tabular}{|l|l|l|}
\hline Level & OCRA Index Values & Risk Level \\
\hline 1 & $<2.2$ & No risk \\
\hline 2 & $2.3-3.5$ & Low risk \\
\hline 3 & $>3.5$ & Risk \\
\hline
\end{tabular}

Table 1: Final Assessment Criteria (OCRA Method)

\section{DHM simulation}

Fig. 2 illustrates the overall simulation development process. In this section, we explain the principles of both our model and the simulation process.

\subsection{Human Model Body and Dynamics}

In our study, the human body was kinematically modelled as a set of articulated rigid bodies branches (Fig. 3), organized into a redundant tree structure, which is characterized by its degrees of freedom (DoF). Depending on the function of the corresponding human segments, each of tree articulation can be modelled into a number of revolute joints. Our DHM therefore comprises 39 joint DoF and 6 root DoF, with 8 DoF for each leg and 7 for each arm. The root is not controlled. Our virtual human models were dimensioned based on subject's anthropometry (Hanavan, 1964).

The dynamics of the robot is described as a second order system as:

$$
M \dot{T}+N T+\gamma=L \tau-J_{e x t}^{t} W_{e x t}
$$

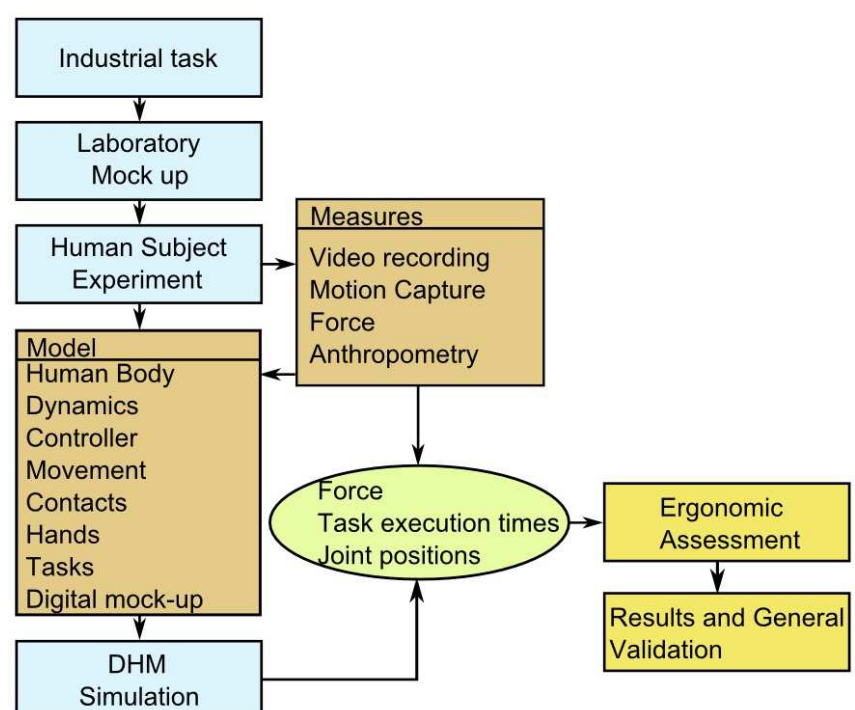

Figure 2: Simulation development process

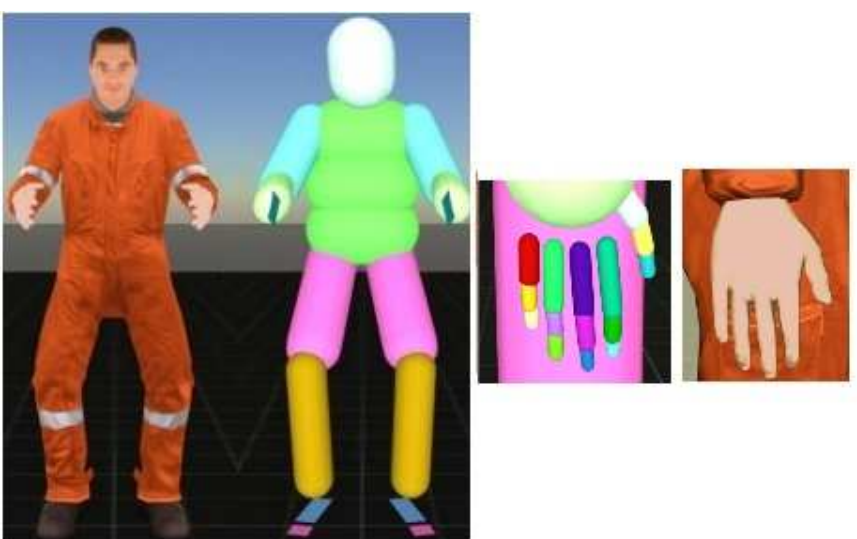

Figure 3: DHM with skinning and collision geometry (left). Right hand model with skinning and collision geometry (right)

in which $M$ is the generalized inertia matrix, $N$ is the matrix of Coriolis and centrifugal forces quadratically dependent upon speed, $\dot{T}$ and $T=\left[V_{\text {root }} \dot{q}_{1} \cdots \dot{q}_{n d o f}\right]^{t}$ are respectively the acceleration and velocity vectors in generalized coordinates. $\gamma$ is the gravity force, $\tau=\left[\tau_{1} \cdots \tau_{n d o f}\right]^{t}$ is the joint torque vector, $L=\left[0_{(n d o f, 6)} I_{n d o f}\right]^{t}$ is the matrix to select the actuated degrees of freedom, $W_{\text {ext }}=[\Gamma F]^{t}$ denotes all the external wrenches (Fig. 5) with $\Gamma$ is the moment and $F$ is the force.

\subsection{Movement computation}

A movement can be characterized, independently of the end-effector, by:

- the initial and final points of the trajectory (position and orientation)

- obstacle positions (via-points of the trajectory)

- duration 
Experimental study of human movements has shown that voluntary movements obey the following three major psychophysical principles:

- Hick-Hyman's law: the average reaction time $T_{R_{a v e}}$ required to choose among probable choices $n$ depends on the logarithm of them (Hyman, 1953):

$$
T_{R_{\text {ave }}}=b \log _{2}(n+1)
$$

- Fitts' law: the movement time depends on logarithm of the relative accuracy (the ratio between movement amplitude and target dimension) (Fitts, 1954):

$$
T_{D}=a+b \log _{2}(2 A W)
$$

where $T_{D}$ is the time, $A$ is the amplitude, $W$ is the accuracy, $a$ and $b$ are empirically determined constants.

- Kinematics invariance: hand movements have a bell-shaped speed profile in straight reaching movements (Morasso, 1981). The speed profile is independent of the movement direction and amplitude. In more complex trajectories (i.e. handwriting) the same principle predicts a correlation between speed and curvature (Morasso and Mussa-Ivaldi, 1982) described as a $2 / 3$ power law:

$$
\dot{s}(t)=c R^{1-\frac{2}{3}}
$$

where $\dot{s}(t)$ is the tangential velocity, $R$ is the radius of curvature and $c$ is a proportionality constant, also termed the "velocity gain factor".

For this reason, more complex trajectories can be divided into overlapping basic trajectory similar to the reaching movements. Such invariant spatio-temporal features of normal movements can be explained by a variety of criteria of maximum smoothness, such as the minimum jerk criterion (Flash and Hogan, 1985) or the minimum torque-change criterion (Uno et al., 1989).

We implemented a modified minimum jerk criterion with via-points to perform trajectories and avoid obstacles.

The original minimum-jerk model may in fact fail to predict the hand path and can only be applied to average data because it predicts a single optimum movement for given via-points. Unlike the original minimum jerk model, the $2 / 3$ power law can be applied to all movements. The main problem with this method is the formula, which predicts speed from paths. In this study, we therefore chose Todorov's model (Todorov and Jordan, 1998), which combines the original and $2 / 3$ power law model and uses a path observed in a specific trial to predict the speed profile. Todorov's model substitutes a smoothness constraint for the $2 / 3$ power law.

In comparison with the $2 / 3$ power law (Eq. (7)), Todorov's model leads to a correlation between speed and curvature:

$$
\dot{s}(t)=A_{s}^{\frac{1}{3}} R^{-\frac{1}{3}}
$$

In the $2 / 3$ power law $A_{s}^{1 / 3}=$ const and $A_{s}^{\prime}=0$. Thus the $2 / 3$ power law is equivalent to setting the normal component of the instantaneous jerk to 0 . In the Todorov's model, $A_{s}$ depends on s(t):

$$
A_{s}=\dot{s}^{3} R(s)
$$

For most of the paths, Todorov model provides trajectories similar to the $2 / 3$ power law, in the sense that they rise and fall concurrently, but are clearly distinguishable.

For a given hand path in space, Todorov's model (Todorov and Jordan, 1998) assumes that the speed profile is the one that will minimize the third derivative of position (or "jerk"):

$$
J=\int_{0}^{T_{D}}\left\|\frac{d^{3}}{d t^{3}} \mathbf{r}[s(t)]\right\|^{2}
$$

with $r(s)=[x(s), y(s), z(s)]$ a $3 \mathrm{D}$ hand path and $s$ is the curvilinear coordinate. In this approach, minimization is performed only over the speed profiles because the path $\mathbf{r}$ is specified.

In the original minimum jerk model (Flash and Hogan, 1985), the minimum jerk trajectory is a 5th-order polynomial in $t$. Using the end-point constraints, we can compute the coefficients of this polynomial. The trajectory and speed are found by a given set of via-points and thus, the hand is constrained to pass through the via-points at definite times. To calculate the minimum jerk trajectory, it is necessary to give passage times $T_{P}$, positions $x$, velocities $v$ and accelerations $a$. In the Todorov's model, the passage times $T_{P}$ are not defined a priori, but are determined by the algorithm explained below.

To find the optimal jerk for any given passage times $T_{P}$ and intermediate points $x$, Todorov's model minimizes the jerk respect $v$ and $a$ by setting the gradient to zero and solving the resulting system of linear equations. To find the intermediate times $T_{P}$, this method uses a nonlinear simplex method to minimize the optimal jerk over all possible passage times.

In the same way as for translations, the speed profile of a rotation is the one that minimizes the third derivative of orientation (or "jerk"), with $r(s)=[\alpha(s), \beta(s), \gamma(s)]$ a $3 D$ rotation path.

In brief, to calculate the minimum jerk trajectory for the rotations and the translations, we need to provide the positions $x$, the initial and final velocities $v$ and the initial and final accelerations $a$. An illustrative example of a minimum jerk trajectory simulation is given in Appendix A.

\subsection{Dynamic DHM control}

We implemented a robotic approach to handle our dynamic DHM: more specifically, we used a joint control. In this kind of robotics context, a common solution is pure stiffness compensation of internal and external disturbances. An alternative solution would be some combination of anticipatory feedforward and feedback control. 
This reasoning is especially suited to human simulation. A number of studies have shown that the central nervous system (CNS) uses internal representations to anticipate the consequences of dynamic interaction forces. In particular, (Lackner and Dizio, 1994) demonstrated that the CNS is able to predict the centripetal and Coriolis forces; (Gribble and Ostry, 1999) demonstrated the compensation of interaction torques during multijoint limb movement. These studies suggest that the nervous system has sophisticated anticipatory capabilities. This is why we need to design accurate internal models of body dynamic and contact.

Generally, a feedforward control model is based on the anticipatory computation of the forces that will be needed by the system to carry out a desired motion plan, without sensory information. It corresponds to solving motion Eq. (4) with respect to the torques generated by the actuators.

An illustrative example is Kawato's feedback error learning model (Kawato and Gomi, 1992), based on cooperation between of two control mechanisms: a feedback loop, which operates in an initial training phase, and the feedforward model, which subsequently emerges. In this model, the feedback error is used as the learning signal for the feedforward model, which gradually takes control any dynamic disturbances, and thereby acquires an internal model of the body dynamics.

We propose a new dynamic DHM controller (Fig. 4), based on this type of combined anticipatory feedforward and feedback control systems based on the notion underlying the acceleration-based control method (Abe et al., 2007)(Colette et al., 2008) and the Jacobian-Transpose (JT) control method (Pratt et al., 1996)(Liu et al., 2011)(DeMagistris et al., 2011). This controller is formulated as two successive Quadratic Programming (QP) problems involving multiple degrees of freedom for simultaneously solving all the constraint equations. The first problem is feedforward control and second is feedback. This computational optimization framework is detailed in Appendix B.

To simulate the task described in Section 2, several objectives have been identified and prioritized:

1. Centre of Mass (com). The dynamic controller maintains the DHM balance by imposing that the horizontal plane projection of the centre of mass (com) lies within a convex support region (Bretl and Lall, 2008).

2. Thorax. During the experimental task, we observed that thorax orientation varied very little. We therefore set the desired thorax orientation equal to its initial orientation.

3. Posture. To obtain more realistic movements and to help the controller to avoid any singularity, we specified one DHM reference joint position for the whole simulation.

4. End effectors (EE). This objective deals with the hand movements required to perform the specific manipulation task.

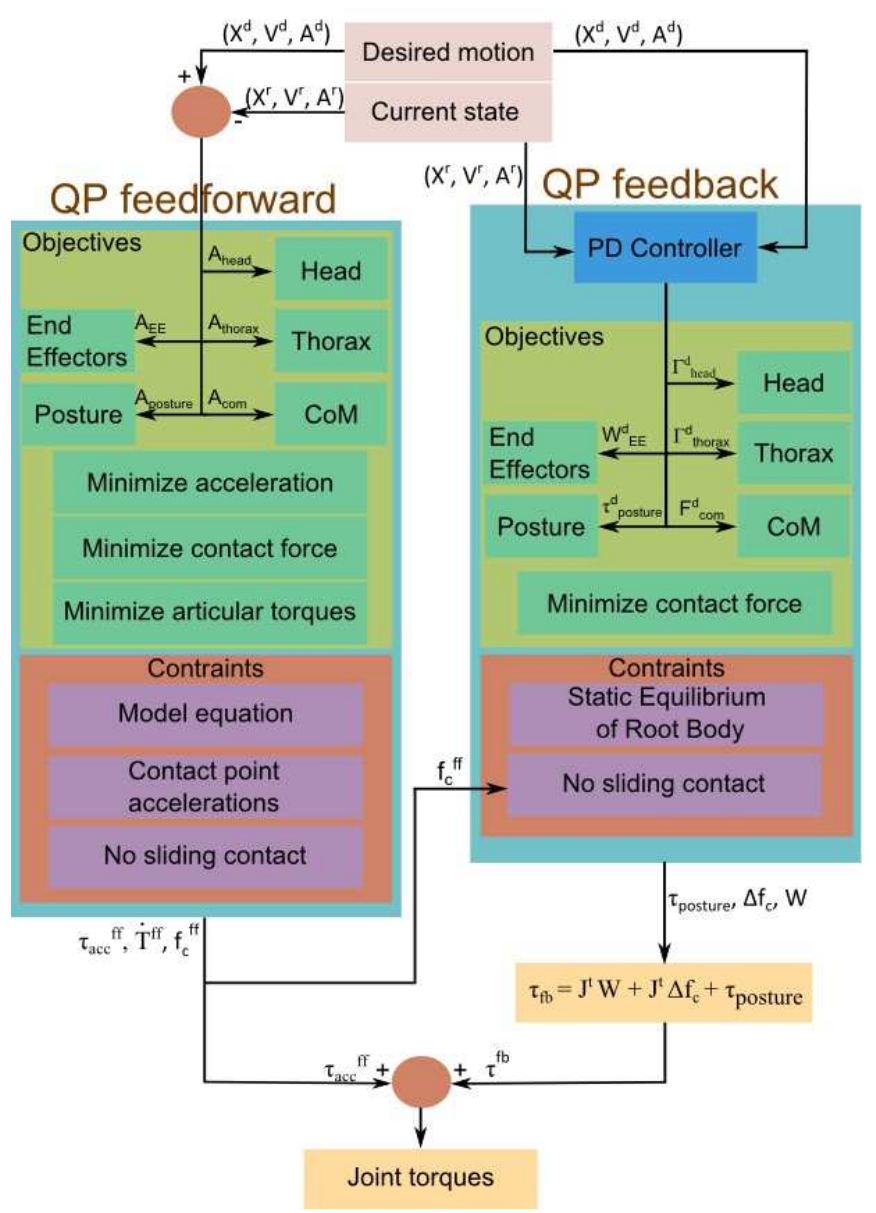

Figure 4: Block diagram of the dynamic DHM control

5. Head. When studying the real work task, we noticed that the head follows the movement of the end effector performing the predominant task. This is the head objective.

6. Contact force. This objective is not a target tracking objective, but it is used to minimize contact force and, as its desired value is unknown a priori, we set the desired contact force to zero.

7. Gravity compensation. This objective is supposed to make target tracking control independent of gravity compensation.

\subsection{Contacts}

Simulations were based on the XDE physics simulation module developed at the CEA-LIST. This module manages the whole physics simulation in real time, including accurate and robust contact detection. Advanced friction effects were modelled in compliance with Coulomb's friction law, which can be formulated as:

$$
\left\|f_{x y}\right\| \leq \mu\left\|f_{z}\right\|
$$

with $\left\|f_{x y}\right\|$ the tangential contact force, $\mu$ the dry friction factor and $\left\|f_{z}\right\|$ the normal contact force. 


\subsection{Hands}

The hand model, illustrated in Fig. 3, has 20 DoF. We use a simple Proportional-Derivative controller to control joint position $\theta$ where a set of desired position $\theta^{d}$ corresponds to open/close hand and different preset grasps.

\subsection{Digital mock-up}

The DMU (Digital mock-up) scenario (Fig. 5) reproduces a virtual environment by ensuring geometric similarity. The inputs used to build the DMU scenario are the workplace spatial organization ( $\mathrm{x}, \mathrm{y}$ and $\mathrm{z}$ dimensions), inserts and tool descriptions ( $\mathrm{x}, \mathrm{y}, \mathrm{z}$ positions and weight) and the DHM position.

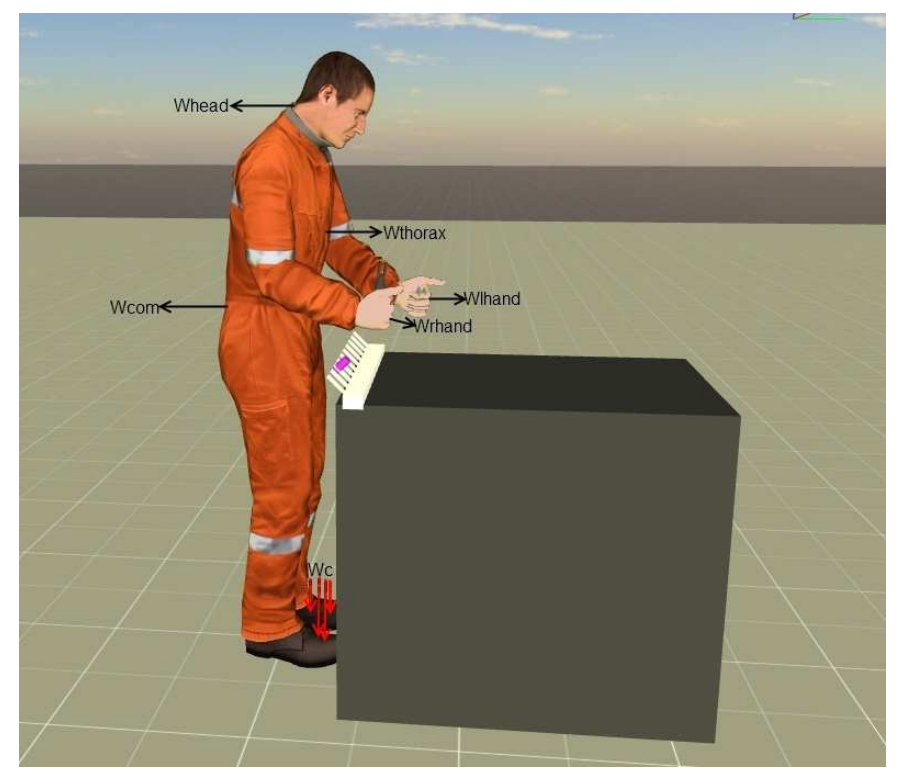

Figure 5: DMU scenario with the desired wrenches: com (centre of mass) for balance, head following end effector (EE) movement, thorax avoiding large movement, c (contacts) for no sliding contacts, lhand (left hand) and rhand (right hand) are end effector tasks when performing handling action

\subsection{Task Models}

It should firstly be stressed that every work task is normally broken up into sequences of a few elementary motions or postures. This is the basics principle underlying many design methods (e.g. Method Time Measurement for instance (Maynard et al., 1948)) or ergonomic assessment index (OCcupational Repetitive Actions (OCRA) (Occhipinti, 1998)).

During our experiments, we noticed that six subjects performed the insert clipping task with the fingers of their two hands and that five subjects performed the same task with the fingers of only one hand. We therefore modelled the two clipping tasks based on three different Finite State Machines (FSM), one for each variant of the insert fitting task using the fingers, and one for the task using a handheld tool.
Ergonomics assessments of both experimental and simulated tasks were based on the OCRA index. According to the OCRA method and vocabulary, the task is broken down into six states, when only the right upper limb is working (Fig. 6):

1. Idle: at the start of the simulation, the DHM body is upright and its arms are along the body.

2. Reach: the right hand adopts a grasping position and the head follows the right hand movement.

3. Grasp: the right hand closes the finger and picks up the insert.

4. Position: the right hand moves to preset insertion point and the head follows the right hand movement.

5. Push: the DHM pushes the insert into the appropriate support with the same force as that measured on the force connected to the table.

6. Release: the right hand fingers open.

7. Idle: the DHM returns to its initial position.

When the right and left upper limbs are working, we have two simultaneous tasks (Fig. 6). When the task is performed with the hand-held tool, the FSM is equal to that for the task performed with two hands, but the RELEASE LHAND GRASP RHAND state is replaced by the RELEASE LHAND POSITION RHAND state.

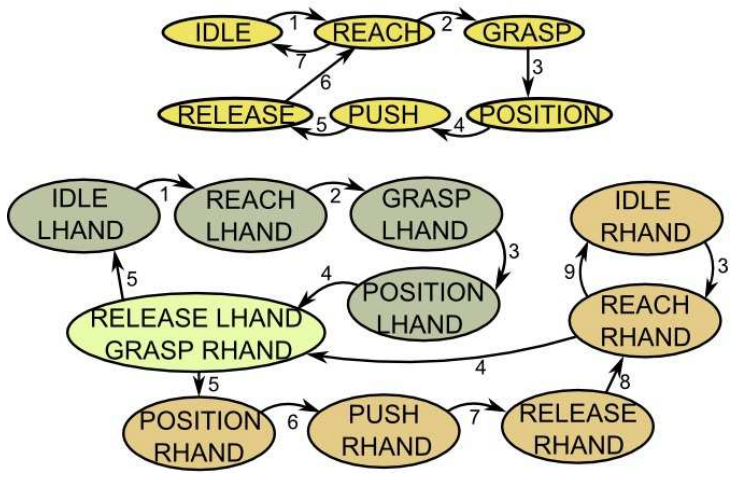

Figure 6: Task modeling for the one-handed (top) and for the twohanded task (bottom)

Table 2 shows the different inputs for the different states.

\section{Results}

Our simulation framework requires a $\mathrm{PC}$ running a Python 2.7 environment with XDE modules. The simulation time step is $0.01 \mathrm{~s}$ and the optimization weights for the different objectives are: $10^{4}$ for com, $10^{3}$ for the tho$\operatorname{rax}, 5 \cdot 10^{3}$ for the right hand task, $10^{1}$ for the posture, $10^{2}$ for the head, $10^{0}$ for the contact task, $10^{2}$ for gravity compensation. These weights are empirically chosen based on the importance and priorities of the different objectives. The same weighting is applied to all simulations; for the two-handed task and when the task is performed with the 


\begin{tabular}{|c|c|}
\hline State & Input \\
\hline REACH & $\begin{array}{l}\text { - duration time } \\
\text { - in presence of obstacle, } \\
\text { intermediate } 6 \mathrm{D} \text { point (via } \\
\text { point to obstacle avoidance) } \\
\text { - final } 6 \mathrm{D} \text { point }\end{array}$ \\
\hline POSITION & $\begin{array}{l}\text { - duration time } \\
\text { - final } 6 \mathrm{D} \text { point }\end{array}$ \\
\hline GRASP & $\begin{array}{l}\text { - duration time } \\
\text { - hand fingers position }\end{array}$ \\
\hline PUSH & $\begin{array}{l}\text { - duration time } \\
\text { - push force }\end{array}$ \\
\hline RELEASE & $\begin{array}{l}\text { - duration time } \\
\text { - hand fingers position }\end{array}$ \\
\hline
\end{tabular}

Table 2: Inputs for the different states of FSM

hand-held tool, a weight of $10^{3}$ is added for the left hand task.

Tables 3, 4 and 5 relate to the comparison between the OCRA index and multiplier parameters (mean \pm standard deviation) based on actual data and DHM simulations for the three different tasks for the Right Upper Limb (RUL) and the Left Upper Limb (LUL): reference data are real forces and recorded joint angles of human subjects and the corresponding simulated forces and joint angles with the respective DHM. The number of subjects in each table corresponds to those who completed the task according to the specified method. Based on experimental observations detailed in Section 2.6, we simulated six subjects for the one-hand-task (Table 3), four subjects for the two-handstask (Table 4) and eleven subjects for the hand-held tool task (Table 5). One subject is not considered in our study.

\section{Discussion}

This study allowed development of a new dynamic DHM controller inspired by human motor control based on robotics and physic simulation. An experimental insertfitting activity was simulated and assessed based on OCRA ergonomic index. This assessment was compared with a real human data-based assessment and consistency was noted.

Our DHM controller has several advantages:

1. The DHM requires minimal information for a simulation: a starting point, an intermediate point for obstacle avoidance and an end point, along with the applied force for insert clipping.

2. Although robotics optimization calculation may lead to extremely high joint torques, our DHM simulations give forces and torques compatible with human performance. For example, the maximum simulated value of the right elbow flexion torque is about $22 N \cdot m$ (respectively $5 N \cdot m$ for the wrist torque, see

\begin{tabular}{|l|c|c|}
\cline { 2 - 3 } \multicolumn{1}{c|}{} & $\begin{array}{c}\text { Human Subject } \\
\text { Experiment }\end{array}$ & DHM \\
\cline { 2 - 3 } \multicolumn{1}{c|}{} & RUL & RUL \\
\hline NTC & 3 & 3 \\
\hline CT & $4.89 \pm 0.41$ & $4.07 \pm 0.43$ \\
\hline F & $0.39 \pm 0.04$ & $0.41 \pm 0.04$ \\
\hline D & 18 & 18 \\
\hline ATA & 30 & 30 \\
\hline CF & $0.64 \pm 0.19$ & $0.64 \pm 0.19$ \\
\hline FoM & $0.55 \pm 0.08$ & $0.52 \pm 0.04$ \\
\hline PoM & 0.7 & 0.7 \\
\hline ReM & 1 & 1 \\
\hline AdM & $2.93 \pm 1.20$ & $2.83 \pm 0.85$ \\
\hline RPA & 1 & 1 \\
\hline DuM & 1 & 1 \\
\hline RcM & $2.93 \pm 1.20$ & $2.83 \pm 0.85$ \\
\hline RTA & $\mathbf{7 . 2 5} \pm \mathbf{3 . 7 0}$ & $\mathbf{7 . 1 9} \pm \mathbf{3 . 3 9}$ \\
\hline OCRA & Risk & Risk \\
\hline Risk Level & & \\
\hline
\end{tabular}

Table 3: OCRA Index Values for the one-hand-task. Mean \pm standard deviation for six subjects

Fig. 7). These values are always smaller than maximum admissible torque at the elbow and wrist joints (this maximum torque is approximately $70 \mathrm{~N} \cdot \mathrm{m}$ for men and $35 N \cdot m$ for women (Askew et al., 1981) at the elbow and approximately $8.05 \mathrm{~N} \cdot \mathrm{m}$ in flexion and $6.53 N \cdot m$ in extension (Ciriello et al., 2001) at the wrist).

This is particularly noticeable since common DHM software may compute joint torques and/or working posture that are not coherent with human performances, thus leading to erroneous ergonomic assessments (Lamkull et al., 2009)(Savin, 2011).

\begin{tabular}{|l|c|c|c|c|}
\cline { 2 - 5 } \multicolumn{1}{c|}{} & \multicolumn{2}{c|}{$\begin{array}{c}\text { Human Subject } \\
\text { Experiment }\end{array}$} & \multicolumn{2}{c|}{ DHM } \\
\cline { 2 - 5 } \multicolumn{1}{c|}{} & LUL & RUL & LUL & RUL \\
\hline NTC & 1 & 3 & 3 & 2 \\
\hline CT & $4.14 \pm 0.14$ & $4.14 \pm 0.14$ & $4.35 \pm 0.13$ & $4.35 \pm 0.13$ \\
\hline F & $14.51 \pm 0.46$ & $43.53 \pm 1.39$ & $13.80 \pm 0.41$ & $41.41 \pm 1.23$ \\
\hline D & $0.41 \pm 0.01$ & $0.41 \pm 0.01$ & $0.44 \pm 0.01$ & $0.44 \pm 0.01$ \\
\hline ATA & 6 & 18 & 6 & 18 \\
\hline CF & 30 & 30 & 30 & 30 \\
\hline FoM & 1 & $0.57 \pm 0.15$ & 1 & $0.57 \pm 0.15$ \\
\hline PoM & $0.63 \pm 0.25$ & $0.53 \pm 0.05$ & $0.63 \pm 0.25$ & $0.55 \pm 0.10$ \\
\hline ReM & 0.7 & 0.7 & 0.7 & 0.7 \\
\hline AdM & 1 & 1 & 1 & 1 \\
\hline RPA & $5.41 \pm 2.10$ & $2.60 \pm 0.73$ & $5.70 \pm 2.23$ & $2.89 \pm 1.01$ \\
\hline DuM & 1 & 1 & 1 & 1 \\
\hline RcM & 1 & 1 & 1 & 1 \\
\hline RTA & $5.41 \pm 2.10$ & $2.60 \pm 0.73$ & $5.70 \pm 2.23$ & $2.89 \pm 1.01$ \\
\hline OCRA & $\mathbf{1 . 2 1} \pm \mathbf{0 . 3 4}$ & $\mathbf{7 . 4 6} \pm \mathbf{2 . 6 2}$ & $\mathbf{1 . 1 5} \pm \mathbf{0 . 3 2}$ & $\mathbf{6 . 9 3} \pm \mathbf{2 . 7 8}$ \\
\hline Risk & \multirow{2}{*}{ No Risk } & Risk & No Risk & Risk \\
Level & & & & \\
\hline
\end{tabular}

Table 4: OCRA Index Values for the two-hands-task. Mean \pm standard deviation for four subjects 


\begin{tabular}{|l|c|c|c|c|}
\cline { 2 - 5 } \multicolumn{1}{c|}{} & \multicolumn{2}{c|}{$\begin{array}{c}\text { Human Subject } \\
\text { Experiment }\end{array}$} & \multicolumn{2}{c|}{ DHM } \\
\cline { 2 - 5 } \multicolumn{1}{c|}{} & LUL & RUL & LUL & RUL \\
\hline NTC & 1 & 3 & 3 & 2 \\
\hline CT & $3.99 \pm 0.37$ & $3.99 \pm 0.37$ & $4.14 \pm 0.35$ & $4.14 \pm 0.35$ \\
\hline F & $30.28 \pm 2.88$ & $30.28 \pm 2.88$ & $29.17 \pm 2.56$ & $29.17 \pm 2.56$ \\
\hline D & $0.40 \pm 0.04$ & $0.40 \pm 0.04$ & $0.41 \pm 0.03$ & $0.41 \pm 0.03$ \\
\hline ATA & 12 & 12 & 12 & 12 \\
\hline CF & 30 & 30 & 30 & 30 \\
\hline FoM & 1 & $0.87 \pm 0.14$ & 1 & $0.87 \pm 0.14$ \\
\hline PoM & $0.61 \pm 0.16$ & $0.52 \pm 0.04$ & $0.61 \pm 0.16$ & $0.51 \pm 0.03$ \\
\hline ReM & 0.7 & 0.7 & 0.7 & 0.7 \\
\hline AdM & 1 & 1 & 1 & 1 \\
\hline RPA & $5.12 \pm 1.43$ & $3.76 \pm 0.74$ & $5.30 \pm 1.44$ & $3.82 \pm 0.61$ \\
\hline DuM & 1 & 1 & 1 & 1 \\
\hline RcM & 1 & 1 & 1 & 1 \\
\hline RTA & $5.12 \pm 1.43$ & $3.76 \pm 0.74$ & $5.30 \pm 1.44$ & $3.82 \pm 0.61$ \\
\hline OCRA & $\mathbf{2 . 4 9} \pm \mathbf{0 . 6 1}$ & $\mathbf{3 . 3 0} \pm \mathbf{0 . 6 7}$ & $\mathbf{2 . 4 0} \pm \mathbf{0 . 5 7}$ & $\mathbf{3 . 2 3} \pm \mathbf{0 . 6 1}$ \\
\hline Risk & Very Low & Very Low & Very Low & Very Low \\
Level & Risk & Risk & Risk & Risk \\
\hline
\end{tabular}

Table 5: OCRA Index Values for the tool-task. Mean \pm standard deviation for all subjects

3. Our controller can solve simulations including non coplanar contacts, friction and dynamics. It achieves the best available robustness to external perturbations. It can solve the whole control problem in one procedure in quasi real time to find the optimal solution with respect to all the considered criteria. For our simulation, computation time is 1.5 times longer than time step.

A first level of robustness of our controller has already been checked. We have changed the anthropometry of the subject and the scenario (e.g. position and orientation of insert final location) and this did not affect the stability or performance of the simulation. Joint torques (Fig. 7), DHM movements (Fig. A.8) and their related OCRA assessment appear realistic and consistent with humanlike behaviour and performance (ergonomic assessment depends on joint angle classes rather than individual continuous values, so light differences of posture or exertion don't modify significantly the assessment). However, a more accurate and quantitative comparison of simulated and real trajectories should be conducted.

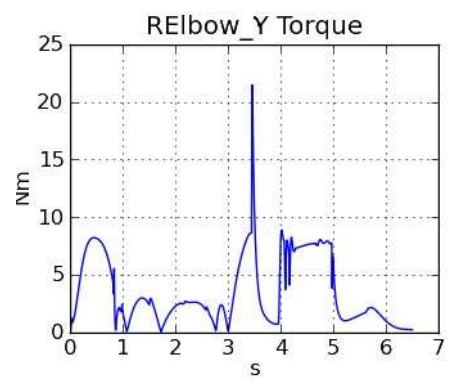

(a) Right elbow torque

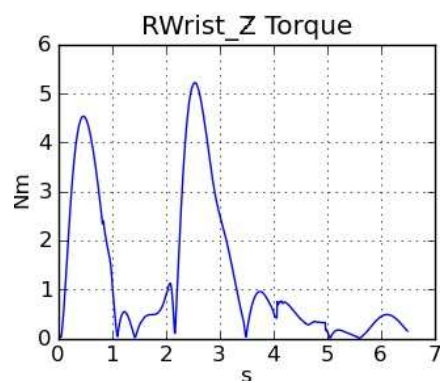

(b) Right wrist torque
Figure 7: Simulated torques are compatible with human performance

\section{Conclusions}

In this paper, we introduced a dynamic digital human model controlled in relation to force and acceleration. We used this model to simulate an experimental insert clipping activity in quasi-real-time and applied the simulated postures, time and exertions to an OCRA index-based ergonomic assessment. Given only scant information on the scenario (typically initial and final operator-positions and clipping force), the simulated ergonomic evaluations were in the same risk area as human data. Although advanced statistics as ANOVA (Analysis of Variance) have not yet been performed for simulations, the mean OCRA indexes (Tables 3, 4 and 5) were in the same risk area as our simulations.

These encouraging results show that a DHM controller may soon overcome the limits of currently common DHM software.

Based on human physiology and simulated training, we wish to improve our controller in the near future to enable it to modulate impact forces and impedance during contact (Burdet et al., 2001)(Yang et al., 2011).

\section{Appendix A. Minimum Jerk simulation}

To illustrate that implemented minimum jerk trajectory respects the kinematics invariance principle, Fig. A.8 illustrates the result of the simulating minimum jerk trajectory. In this example, the right hand trajectory is arbitrarily defined by the following right hand positions (starting and ending points, three intermediate points):

$$
\begin{aligned}
X^{\text {initial }} & =[0.26,0.01,0.86,-0.50,-0.53,-0.51,0.45] \\
X^{\text {int }} & =[0.31,0.11,0.92,-0.50,-0.53,-0.51,0.45] \\
& =[0.36,0.06,0.94,-0.50,-0.53,-0.51,0.45] \\
& =[0.26,0.01,0.86,-0.50,-0.53,-0.51,0.45] \\
X^{\text {final }} & =[0.36,0.16,0.96,-0.50,-0.53,-0.51,0.45]
\end{aligned}
$$

with a movement duration of $T_{D}=1.3 \mathrm{~s}$. The first three values of $X$ are the right hand translation vector and its four subsequent values are the quaternion representing the right hand rotation.

\section{Appendix B. Optimization framework}

We introduce the following notation: $Z$ is the unknown vector, $Z^{d}$ is the desired, but not necessarily accessible solution, $Q$ is the symmetric matrix describing the coefficients of the quadratic terms and $A, b, N, d$ are matrices and vectors expressing the linear equality and inequality constraints.

$$
\min \frac{1}{2}\left\|Z-Z^{d}\right\|_{Q}^{2} \quad\left\{\begin{array}{l}
A \cdot Z+b=0 \\
N \cdot Z+d \geq 0
\end{array}\right.
$$

The controller is introduced to compute joint torques that achieve different objectives and satisfies different constraints. The unknown vector $\mathrm{Z}$ is:

$$
Z=\left[\begin{array}{lll}
\tau & \dot{T} & f_{c}
\end{array}\right]^{t}
$$



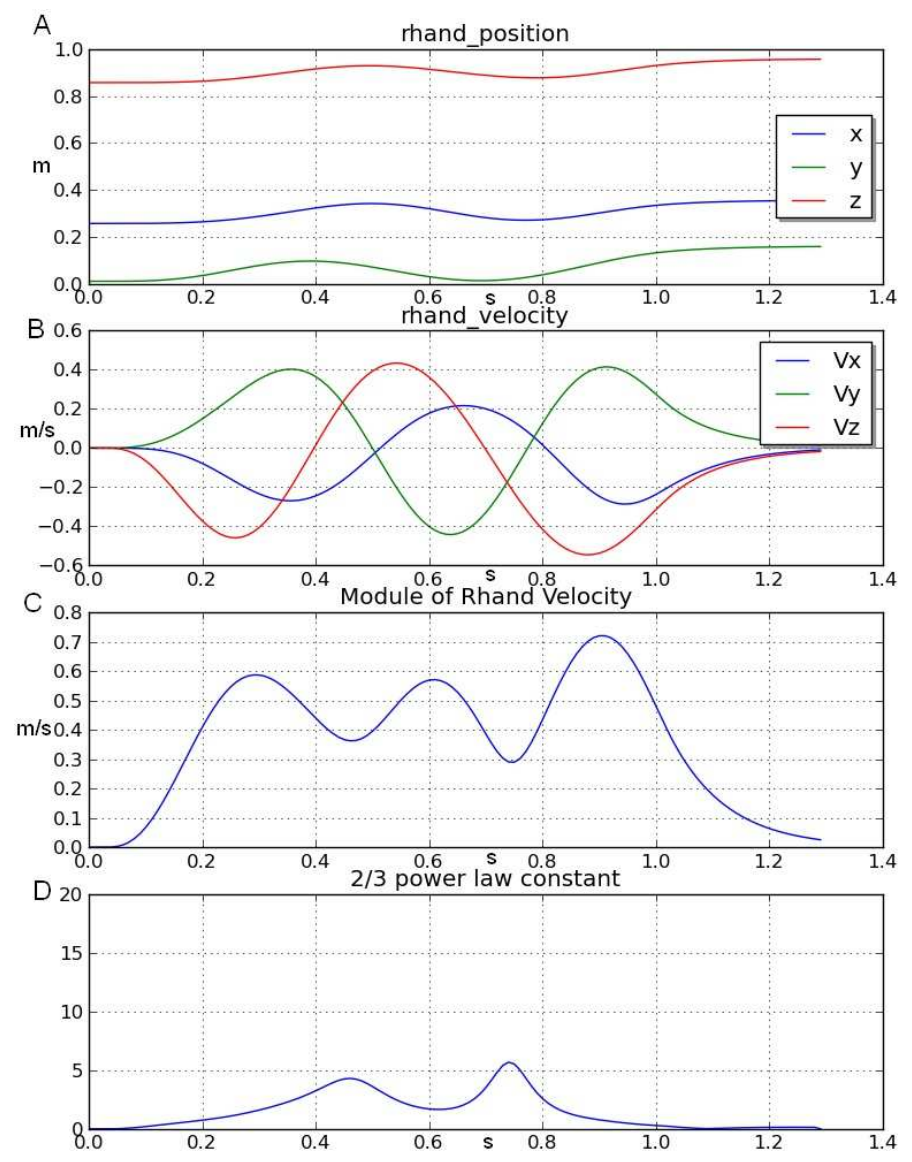

Figure A.8: Minimum jerk simulation. XYZ positions (A) and velocities (B); the velocity modules (C); the constant $c$ of the Eq. (7) to validate that the implemented minjerk model respects the $2 / 3$ power law (D)

\section{Appendix B.1. Acceleration feedforward $Q P$}

During the feedforward phase, the objectives are:

1. Objective based on acceleration control. Control is based on a acceleration control method adapted to fulfill task objectives such as the tracking of desired com position (com), thorax orientation, head orientation and end effector (EE) movements. The goal is to minimize the difference between the actual acceleration $a$ and desired acceleration $a^{d}$ founded by the minimum jerk optimization. The desired acceleration does not depend on the motion error, but only of the planned acceleration (feedforward).

$a$ is expressed in terms of the unknowns of the system $\dot{T}$ as:

$$
\left\{\begin{array}{l}
v=J T \\
a=J \dot{T}+\dot{J} T
\end{array}\right.
$$

2. Minimize torques. We set the desired torques to zero. $\tau_{a c c}^{d}=0_{\left(n_{\text {dof }}, 1\right)}$

3. Minimize contact forces. We want to minimize $f_{c}$, so $f_{c}^{d}$ is set to zero, as it is unknown a priori.
4. Minimize accelerations. We set the desired accelerations to zero. $\dot{T}^{d}=0_{\left(6+n_{d o f}, 1\right)}$

During the feedforward phase, the constraints are:

1. Dynamic equation. The dynamic equation in Eq. 4 is expressed as a function of the unknown $\mathrm{Z}$ :

$$
\left[\begin{array}{lll}
-L & M & J_{c}^{t}
\end{array}\right] Z+(\gamma+N T)=0
$$

2. Contact point accelerations. To help maintain contacts, contact acceleration must be null.

$$
a_{c}=J_{c} \dot{T}+\dot{J}_{c} T=0
$$

The constraint on the contact point accelerations expressed as a function of the unknown $\mathrm{Z}$ is:

$$
\left[\begin{array}{lll}
0 & J_{c} & 0
\end{array}\right] Z+\left(\dot{J}_{c} T-a_{c}^{d}\right)=0
$$

3. No-sliding contacts. The no-sliding contacts are expressed as a set of inequality constraints. Contact constraints are imposed at the contact points between the foot and the ground. The contact force $f_{c_{j}}$ should remain within the friction cone. We apply a linearized Coulomb friction model, in which the friction cone of each contact is approximated by a $n$ faced polyhedral convex cone. The contact constraints are formularized as:

$$
E_{c_{i}} f_{c_{i}}+d_{c_{i}}<0
$$

where $E_{c_{i}}$ is the approximated friction cone, $d_{c_{i}}$ is a customer defined margin vector, so that the projection of $f_{c_{i}}$ on the normal vector of each facet of the friction cone should be kept larger than $d_{c_{i}}$.

The constraint on the contact forces expressed as a function of the unknown $\mathrm{Z}$ is:

$$
\left[\begin{array}{lll}
0 & 0 & E_{c}
\end{array}\right] Z+d_{c}<0
$$

We summarize the feedforward phase as:

$$
\hat{O}=\underset{\substack{a \\
a_{\text {com }}, a_{E E}, a_{\text {thorax }}, f_{c_{j},}, \dot{T}}}{\arg \min \frac{1}{2}}\left\|\left[\begin{array}{c}
a_{\text {com }} \\
a_{E E} \\
a_{\text {head }} \\
a_{\text {thorax }} \\
\tau_{\text {acc }} \\
\dot{T} \\
f_{c_{j}}
\end{array}\right]-\left[\begin{array}{c}
a_{c o m}^{d} \\
a_{E E}^{d} \\
a_{\text {head }}^{d} \\
a_{\text {thorax }}^{d} \\
\tau_{a c c}^{d} \\
\dot{T} d \\
f_{c_{j}}^{d}
\end{array}\right]\right\|_{Q}^{2}
$$

Subject to:

$$
\left\{\begin{array}{l}
M \dot{T}+N T+\gamma=L \tau-J_{c}^{t} f_{c} \\
E_{c} f_{c}+d_{c} \geq 0 \\
J_{c} \dot{T}+\dot{J}_{c} T=0
\end{array}\right.
$$

With this optimization, we obtain $\tau_{a c c}^{f f}, f_{c}^{f f}, \dot{T}^{f f}$. 
Appendix B.2. Force feedback $Q P$

The unknown vector $\mathrm{Z}$ is:

$Z=\left[\begin{array}{llllll}W_{E E} & F_{\text {com }} & \Gamma_{\text {thorax }} & \Gamma_{\text {head }} & \tau_{\text {posture }} & \Delta f_{c}\end{array}\right]^{t}$

During the feedback phase the objectives are:

1. Force control-based. This control is introduced to correct the movement on the fly. Furthermore, it allow us to handle contacts during the manipulation by setting the desired contact forces. Given a desired wrench $W^{d}$ in the Cartesian coordinate space, the equivalent joint torques $\tau$ can be obtained by means of the principle of the virtual works $\tau=J^{t} W^{d}$, with $J$ the Jacobian matrix at the point where $W^{d}$ is supposed to be applied. The goal of our control system is to compute joint torques based on given tasks. For each task, we imagine that a virtual wrench $W^{d}$ is applied at a certain point on the body of the DHM to guide its motion (Fig. 5). We define the desired wrench using a proportional-derivative $(\mathrm{PD})$ feedback control law:

$$
W^{d}=K \delta\left(H^{d}, H^{r}\right)+D \delta\left(V^{d}, V^{r}\right)+W^{f f}
$$

with $H^{r} \in S E(3), H^{d} \in S E(3), V^{r} \in \operatorname{se}(3)$ and $V^{d} \in$ se(3), where $S E(3)$ is the special Euclidean group and $s e(3)$ is the Lie algebra of $S E(3) . \delta\left(H^{d}, H^{r}\right)$ stands for error between the desired and the current position and orientation, while $\delta\left(V^{d}, V^{r}\right)$ stands for error between the desired and the current linear and angular velocities. $K$ and $D$ are the proportional and derivative gain matrix respectively. For the com task only the position error is considered, while for thorax and head tasks only the orientation error are considered. An additional $W^{f f}$ can be added, if a contact is established the end effector and an object during the handling.

This anticipatory feedforward force helps to improve contact between the end effector and the object. It can be defined based on the physical characteristic of the object, such as the weight and the friction coefficient.

2. Posture. The difference between the actual joint torque $\tau_{\text {posture }}$ and the desired joint torque $\tau_{\text {posture }}^{d}$ is minimized. $\tau_{\text {posture }}^{d}$ can be written as:

$$
\tau_{\text {posture }}^{d}=K e+D \dot{e}
$$

with $e$ joint position error and $\dot{e}$ joint velocity error.

3. Minimize contact forces. $\Delta f_{c}^{d}=0_{\left(3 n_{f_{c}}, 1\right)}$ with $Q_{\Delta f_{c}}=w_{\Delta f_{c}} I_{3 n_{f_{c}}}$

During feedback phase the constraints are:

1. Static equilibrium of rigid body mode. The wrenches are constrained by the static equilibrium of the root body:

$$
\begin{aligned}
& 0=J_{\text {com }}^{\text {root }}{ }^{t} F_{\text {com }}+J_{\text {thorax }}^{\text {root }}{ }^{t} \Gamma_{\text {thorax }}+J_{\text {head }}^{\text {root }}{ }^{t} \Gamma_{\text {head }} \\
& +J_{E E}^{\text {root }}{ }^{t} W_{E E}+J_{c}^{\text {root }}{ }^{t} \Delta f_{c_{i}}
\end{aligned}
$$

\section{No-sliding contact}

$$
E_{c}\left(f_{c}^{f f}+\Delta f_{c}\right)+d_{c} \geq 0
$$

We summarize the feedback phase as:

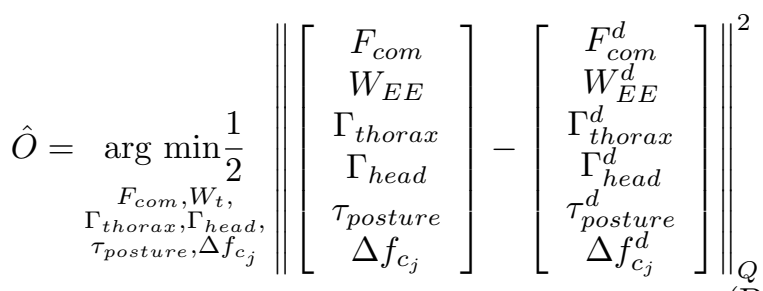

Subject to:

$$
\left\{\begin{array}{l}
0=J_{\text {com }}^{\text {root }}{ }^{t} F_{\text {com }}+J_{\text {thorax }}^{\text {root }}{ }^{t} \Gamma_{\text {thorax }}+J_{\text {thorax }}^{\text {root }}{ }^{t} \Gamma_{\text {head }} \\
+J_{E E}^{\text {root }}{ }^{t} W_{E E}+J_{c}^{\text {root }}{ }^{t} \Delta f_{c_{i}} \\
E_{c}\left(f_{c}^{f f}+\Delta f_{c}\right)+d_{c} \geq 0
\end{array}\right.
$$

The feedback joint torque can be obtained by:

$$
\begin{aligned}
& \tau^{f b}=J_{\text {com }}^{t} F_{\text {com }}+J_{\text {thorax }}^{t} \Gamma_{\text {thorax }}+J_{\text {head }}^{t} \Gamma_{\text {head }} \\
& +J_{E E}^{t} W_{E E}+J_{c}^{t} \Delta f_{c}+\tau_{\text {posture }}
\end{aligned}
$$

At the end of the feedforward and feedback phases we obtain respectively $\tau^{f f}$ and $\tau^{f b}$. The joint torques applied to the DHM is equal to:

$$
\tau=\tau^{f f}+\tau^{f b}
$$

\section{References}

Abe, Y., da Silva, M., Popović, J., 2007. Multiobjective control with frictional contacts. In: Proc. ACM SIGGRAPH/EG Symposium on Computer Animation. Aire-la-Ville, Switzerland, pp. 249-258.

AFNOR, 2003. Nf en 1005-2 safety of machinery human physical performance - part 2: Manual handling of machinery and components parts of machinery. AFNOR (La Plaine St-Denis).

AFNOR, 2007. Nf en 1005-5 safety of machinery human physical performance - part 5: Risk assessment for repetitive handling at high frequency. AFNOR (La Plaine St-Denis).

AFNOR, 2008. Nf en 1005-5 - safety of machinery - human physical performance - part 4: Evaluation of working postures and movements in relation to machinery. AFNOR (La Plaine St-Denis).

Askew, L. J., An, K. N., Morrey, B. F., Chao, E. Y., 1981. Functional evaluation of the elbow. normal motion requirements and strength determinations. Orthop. Trans., 5-304.

Badler, N. I., 1997. Virtual humans for animation, ergonomics, and simulation. In: Proceedings of the IEEE workshop on non-rigid and articulates motion. pp. 28-36.

Bernard, B. P., 1997. Musculoskeletal Disorders and Workplace Factors. A Critical Review of Epidemiologic Evidence for WorkRelated Musculoskeletal Disorders of the Neck, Upper Extremity, and Low Back. NIOSH.

Bradwell, B., Li, B., 2008. A tutorial on motion capture driven character animation. In: Eight IASTED International Conference Visualization, Imagin, and Image Processing. Palma de Mallorca.

Bretl, T., Lall, S., 2008. Testing static equilibrium for legged robots. IEEE Transactions on Robotics 24, 794-807.

Burdet, E., Osu, R., Franklin, D. W., Milner, T. E., Kawato, M., 2001. The central nervous system stabilizes unstable dynamics by learning optimal impedance. Nature 414, 446-449. 
CEN, 2008. Safety of machinery - anthropometric requirements for the design of workstations at machinery. European standard EN ISO 14738:2008.

Chaffin, D. B., 2005. Improving digital human modelling for proactive ergonomics in design. Ergonomics 48, 478-491.

Chaffin, D. B., 2007. Human motion simulation for vehicle and workplace design. Human Factors and Ergonomics in Manufacturing $17,475-484$

Ciriello, V. M., Snook, S. H., Webster, B. S., Dempsey, P., 2001. Psychophysical study of six hand movements. Ergonomics 44, 922936

Colette, C., Micaelli, A., Andriot, C., Lemerle, P., 2008. Robust balance optimization control of humanoid robots with multiple non coplanar grasps and frictional contacts. In: Proceedings of the IEEE International Conference on Robotics and Automation. Pasadena, USA, pp. 3187-3193.

DeMagistris, G., Micaelli, A., Andriot, C., Savin, J., Marsot, J., 2011. Dynamic virtual manikin control design for the assessment of the workstation ergonomy. In: First International Symposium on Digital Human Modeling. Lyon.

Dukic, T., Ronang, M., Christmansson, M., 2007. Evaluation of ergonomics in a virtual manufacturing process. Journal of Engineering Design 18, 125-137.

EU, 2006. Eu directive on machinery - directive 2006/42/ec of the european parliament and of the council of 17 may 2006 on machinery. Official Journal of the European Union.

URL http://eur-lex.europa.eu/LexUriServ/LexUriServ.do? uri=0J :L : 2006: 157:0024:0086:EN :PDF

Fitts, P. M., 1954. The information capacity of the human motor system in controlling the amplitude of movement. Journal of Experimental Pshychology 47, 381-391.

Flash, T., Hogan, N., 1985. The coordination of arm movements: an experimentally confirmed mathematical model. Journal of Neuroscience $7,1688-1703$.

Gaudez, C., 2008. Upper limb musculo-skeletal disorders and insert fitting activity in automobile sector: impact on muscular stresses of fitting method and insert position on part. In: Computer Methods in Biomechanics and Biomedical Engineering. Vol. 11. pp. 101-102.

Gomes, S., Sagot, J. C., Koukam, A., Leroy, N., 1999. Manercos, a new tool providing ergonomics in a concurrent engineering design life cycle. In: 4th Annual Scientific Conference on Web Technology, New Media, communications and Telematics. Theory, Methods, Tools and Applications, EUROMEDIA 99. Munich, pp. 237 241

Gribble, P. L., Ostry, D. J., 1999. Compensation for interaction torques during single and multijoint limb movement. Journal of Neurophysiology 82, 2310-2326.

Hanavan, E. P., 1964. A mathematical model of the human body. Wright-Patterson Air Force Base Report No. AMRL-TR-102, 64102 .

Hignett, S., MacAtamney, L., 2000. Rapid entire body assessment (reba). Applied Ergonomics 31, 201-205.

Hyman, R., 1953. Stimulus information as a determinant of reaction time. Journal of Experimental Psychology 45, 188-196.

Kao, S. Y., 2003. Carpal tunnel syndrome as an occupational disease. Journal of the American board of family medicine 16, 533-542.

Kawato, M., Gomi, H., 1992. A computational model of four regions of the cerebellum based on feedback error learning. Biological Cybernetics 69, 95-103.

Lackner, J. R., Dizio, P., 1994. Rapid adaptation to coriolis force perturbations of arm trajectory. Journal of Neurophysiology 72, 299-313.

Lamkull, D., Hanson, L., Ortengren, R., 2009. A comparative study of digital human modelling simulation results and their outcomes in reality: A case study within manual assembly of automobiles. International Journal of Industrial Ergonomics 39, 428-441.

Liu, M., Micaelli, A., Evrard, P., Escande, A., Andriot, C., 2011. Interactive dynamics and balance of a virtual character during manipulation tasks. In: IEEE International Conference on Robotics and Automation. Shanghai, China, pp. 1676-1682.
MacAtamney, L., Cortlett, E. N., 1993. Rula: a survey method for the investigation of work-related upper limb disorders. Applied Ergonomics 24, 91-99.

Maynard, H. B., Stegemerten, G. J., Schwab, J. L., 1948. Methodstime measurement. McGraw Hill book company.

Morasso, P., 1981. Spatial control of arm movements. Experimental Brain Research 42, 223-227.

Morasso, P., Mussa-Ivaldi, F. A., 1982. Trajectory formation and handwriting: a computational model. Biological Cybernetics 45, 131-142.

NST-n168, 1998. Ergonomie des outils à main - Problématique et état de l'art. Note scientifique et technique de l'INRS.

Occhipinti, E., 1998. Ocra, a concise index for the assessment of exposure to repetitive movements of the upper limbs. Ergonomics 41, 1290-1311.

Porter, J. M., Case, K., Marshall, R., Gyi, D., Sims, R., 2004. Beyond jack and jill: designing for individuals using hadrian. International Journal of Industrial Ergonomics 33, 249-264.

Pratt, J., Torres, A., Dilworth, P., Pratt, G., 1996. Virtual actuator control. In: IEEE International Conference on Intelligent Robots and Systems. pp. 1219-1226.

Savin, J., August 2011. Digital human manikins for work-task ergonomic assessment: which degree of confidence and using constraints? Proceedings of the Institution of Mechanical Engineers, Part B: Journal of Engineering Manufacture 225, 1401-1409.

Sjogaard, G., Sejersted, O. M., Winkel, J., Smolander, J., Jorgensen, K., Westgaard, R., 1995. Exposure assessment and mechanisms of pathogenesis in work-related musculoskeletal disorders: Significant aspects in the documentation of risk factors. Work and Health. Scientific Basis of Progress in the Working Environment, edited by O. Svane and C. Johansen.

Todorov, E., Jordan, M. I., 1998. Smoothness maximization along a predefined path accurately predicts the speed profiles of complex arm movements. Journal of Neurophysiology 80, 697-714.

Uno, Y., Kawato, M., Suzuki, R., 1989. Formation and control of optimal trajectory in human multijoint arm movement: Minimum torque-change model. Biological Cybernetics 61, 89-101.

Yang, C., Ganesh, G., Haddadin, S., Parusel, S., Albu-Schaeffer, A., Burdet, E., 2011. Human like adaptation of force and impedance in stable and unstable interactions. Transactions on Robotics 27, 918-930. 